\title{
A high-repetition rate ELM replication system for the Magnum-PSI and Pilot-PSI linear devices
}

\author{
T.W. Morgan ${ }^{1}$ T.M. de Kruif ${ }^{1,2}$ H.J. van der Meiden ${ }^{1}$ M.A. van \\ den Berg ${ }^{1}$ J. Scholten ${ }^{1}$ W. Melissen ${ }^{1}$ B. J. M. Krijger ${ }^{1}$ S. \\ Bardin $^{1}$ and G. De Temmerman ${ }^{1}$ \\ ${ }^{1}$ FOM DIFFER, Dutch Institute for Fundamental Energy Research, Association \\ EURATOM-FOM, Partner in the Trilateral Euregio Cluster, The Netherlands \\ ${ }^{2}$ Fusion Science and Technology Group, Eindhoven University of Technology, \\ Eindhoven, The Netherlands
}

\begin{abstract}
.
High power ELM striking onto divertor components presents one of the strongest lifetime and performance challenges for plasma facing components in future fusion reactors. A high repetition-rate ELM replication system has been constructed and was commissioned at the Magnum-PSI linear device to investigate the synergy between steady state plasma exposure and the large increase in heat and particle flux to the plasma facing surface during repeated ELM transients in conditions aiming to mimic as closely as possible those in the ITER divertor. This system is capable of increasing the electron density and temperature from $\sim 1 \times 10^{20}$ to $\sim 1 \times 10^{21} \mathrm{~m}^{-3}$ and from $1-5 \mathrm{eV}$ respectively, leading to a heat flux increase at the surface up to $\sim 130 \mathrm{MW}$ $\mathrm{m}^{-2}$. By combining Thomson scattering measurements with heat fluxes determined using the THEODOR code the sheath heat transmission factor during the pulses was determined to be $\approx 7.7$, in agreement with sheath theory. The heat flux is found to be linearly dependent upon the strength of the magnetic field at the target position and, by adapting the system to Pilot-PSI, tests at $1.6 \mathrm{~T}$ showed heat fluxes of $>600$ $\mathrm{MW} \mathrm{m}{ }^{-2}$. This gives confidence that with the installation of a $2.5 \mathrm{~T}$ superconducting magnetic solenoid at Magnum-PSI the heat flux will reach the ITER-relevant $\mathrm{GW} \mathrm{m}^{-2}$ heat flux regime.
\end{abstract}

PACS numbers: 52.50.Dg, 52.40.Hf, 52.40.Kh, 52.50.Nr 


\section{Introduction}

The lifetime and heat removal capability of the ITER divertor and those of future highperformance fusion devices remains one of the most crucial questions to address on the path to fusion power. During normal operation the ITER divertor is expected to receive a steady heat and particle flux of $\sim 10 \mathrm{MW} \mathrm{m}^{-2}$ and $\sim 10^{24} \mathrm{~m}^{-2} \mathrm{~s}^{-1}$ [1] . Under such fluxes strong modification of the plasma-exposed surface can occur such as nanostructure formation under helium bombardment [4, 5] and blistering under hydrogen and deuterium plasma exposure [6, 7]. In combination with this edge localised modes (ELMs) will add short periodic bursts of heat and particles. For type-I ELMs power densities of up to $1-10 \mathrm{GW} \mathrm{m}^{-2}$ with a duration between 0.5 and $1 \mathrm{~ms}$ and frequency $1-10 \mathrm{~Hz}$ are expected in ITER [8]. These conditions are not replicated in current tokamak devices, so that their study requires specialised laboratory experiments. Combined experiments with steady state linear plasmas and pulsed high power laser irradiations at NAGDISII [9] and PISCES-A [10] show a reduced damage threshold for tungsten, compared to ELM-replication experiments alone. This suggests a synergistic effect between the steady plasma flux and the transient loading of the surface. This is also supported by experiments in Pilot-PSI using a capacitor bank discharged through the plasma source to produce a transient pulse of not only high heat flux but also high particle fluxes which well replicated the combined steady and transient conditions expected in the ITER divertor [11, 12]. The success of these experiments motivated the development of an improved system which could be used for both the Magnum-PSI and Pilot-PSI linear devices, designed as a Multi-pulse ELM Replication System (MERS). The main innovations are a high repetition rate $(>100 \mathrm{~Hz})$, compared to the previous singlepulse per discharge system, and flexible pulse shape through varying the pulse timing and voltage of different independently controlled capacitors. The development and characterisation of this system as well as initial results will be presented in this work.

\section{Experimental set-up}

Due to its superior diagnostic capabilities and flexibility the Magnum-PSI device was chosen for the commissioning and characterization of the MERS system. Magnum-PSI utilises a cascaded arc discharge [13, 14] confined into a narrow beam (FWHM $\sim 25$ $\mathrm{mm}$ ) by magnetic coils producing up to $1.3 \mathrm{~T}$ which impacts the target $\sim 1 \mathrm{~m}$ from the source, as described in detail in [15] (figure 1). At the target position the machine produces a low temperature $\left(T_{e} \sim 1-3.5 \mathrm{eV}\right)$, high density $\left(n_{e} \sim 1-7 \times 10^{20} \mathrm{~m}^{-3}\right.$ ) plasma with a particle and heat flux of $\Gamma \sim 1 \times 10^{24} \mathrm{~m}^{-2} \mathrm{~s}^{-1}$ and $\leq 12 \mathrm{MW} \mathrm{m}^{-2}$ respectively [16, 17]; conditions which well replicate those expected in the ITER divertor. The surface temperature was monitored using a fast-framing infra-red camera (FLIR $\mathrm{SC} 7500 \mathrm{MB}$ ) used at $\sim 5 \mathrm{kHz}$, while the plasma density and electron temperature were determined via Thomson scattering (section 4).

The cascaded arc source operates with a current typically between $100 \mathrm{~A}$ and 


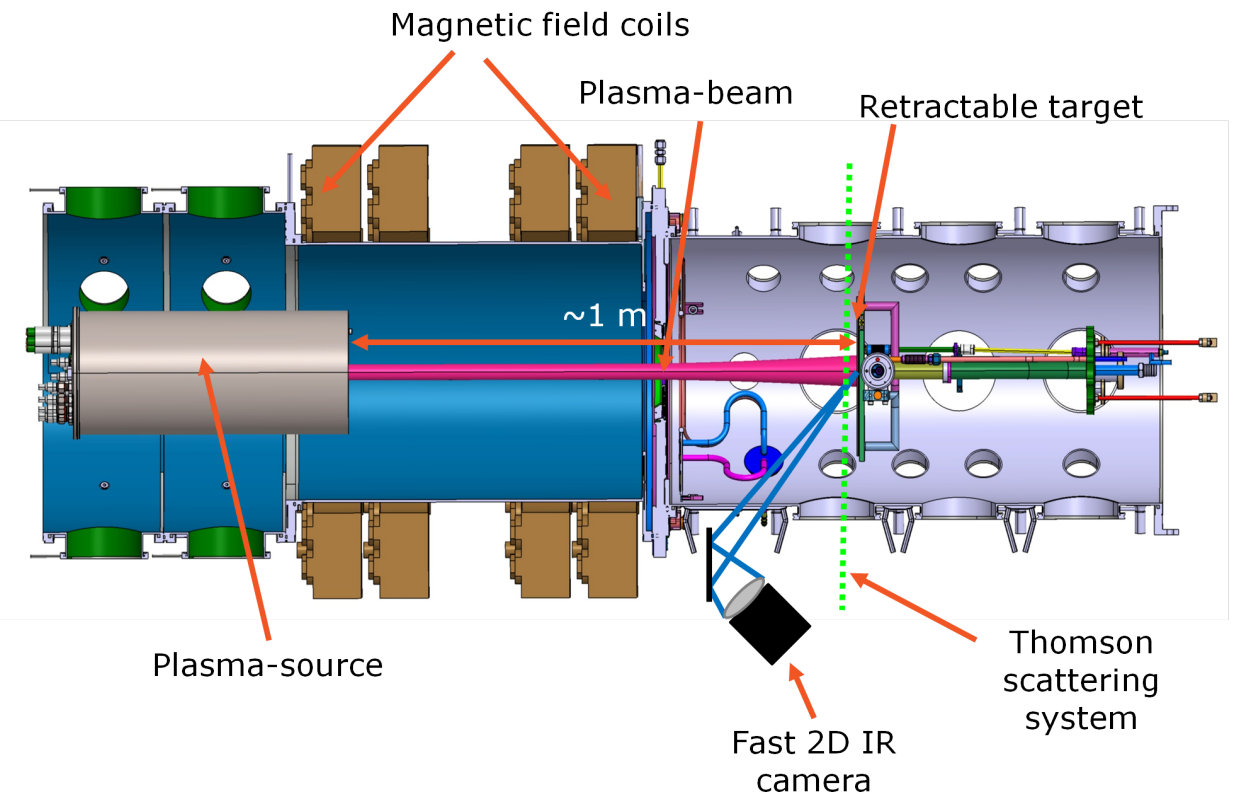

Figure 1: Schematic of the Magnum-PSI device showing the positioning of the Thomson scattering and fast-framing infra-red camera.

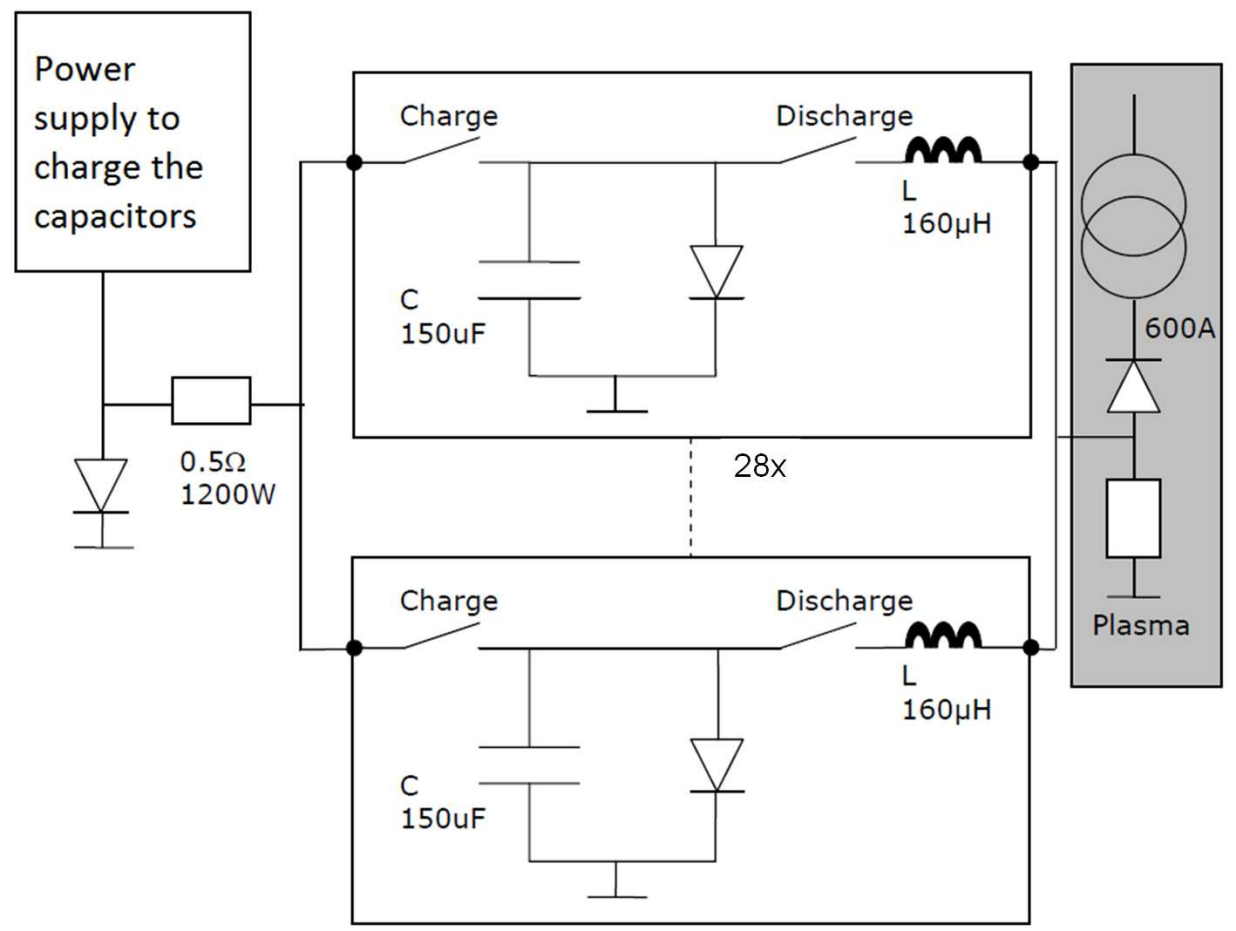

Figure 2: Schematic picture of the capacitor bank system used in Magnum-PSI. 
200 A from a DC current-regulated source. In parallel with this a modular capacitor bank system was installed. This consists of 28 identical sections as shown in figure 2. Each section consists of an inductor $(160 \mu \mathrm{H})$, a capacitor $(150 \mu \mathrm{F})$, externally triggered charging and discharging switches, and an anti-reversal diode. After the capacitor has been charged to the required voltage the stored energy can be dissipated through the plasma's resistance, leading to both Ohmic heating and increased ionisation, and transiently producing higher temperatures and densities. Each capacitor can be charged up to $3000 \mathrm{~V}$, with a total maximum stored energy of $\sim 19 \mathrm{~kJ}$ for the entire system. Each section can be charged and discharged independently with a charging time of $22.2 \mathrm{~ms}$, permitting high repetition rates $>100 \mathrm{~Hz}$ to easily be achieved by discharging in sequence. Further, by varying the delay time between the discharges of several capacitors, the rise time can be changed and the pulse shaped. Alternatively, the total energy of the pulse can be increased by discharging several capacitor banks simultaneously. Therefore a combination of steady state plasma and transient pulses of increased heat and particle flux is created, analogous to the situation expected in the ITER divertor.

The cascaded arc source used was designed to accommodate the high power densities of the transient pulses by using a wide channel diameter $(\oslash 10 \mathrm{~mm})$ lined with molybdenum inserts to reduce impurity production as described in [12]. Due to the increased average heat flux to the nozzle due to repeated pulsing further protection of its surface was required to protect it from melting and impurity production. Therefore the nozzle plate was changed from $\mathrm{Cu}$ to a $\mathrm{CuW}$ alloy (25:75) coated with a $200 \mathrm{~nm}$ thick W layer. This thickness was chosen to avoid flaking, while still maintaining a good coating lifetime.

The current pulse through the source can be described in two modes- the resonance mode and the decay mode. In the resonance mode the anti-reversal diode is nonconducting and the discharge through the plasma forms an RLC circuit. As the circuit is highly under damped $(\zeta<0.1)$ the resulting current curve can be described by the first quarter of a sine wave,

$$
I(t)=I_{0} \sin \left(\frac{t}{\sqrt{L C}}\right) \text { for } 0<t<\frac{\pi}{2} \sqrt{L C},
$$

where $I_{0}$ is the peak current of the system and $L$ and $C$ the total inductance and capacitance of the system, respectively.

The decay mode occurs when the current is at its maximum and the voltage over the capacitors is zero. At this point, the anti-reversal diode becomes conducting and the section functions as an LR circuit. The resulting current through the plasma source then becomes

$$
I(t)=I_{0} e^{-\frac{R t}{L}} \quad \text { for } t>\frac{\pi}{2} \sqrt{L C},
$$

where $R$ is the total resistance of the system. 


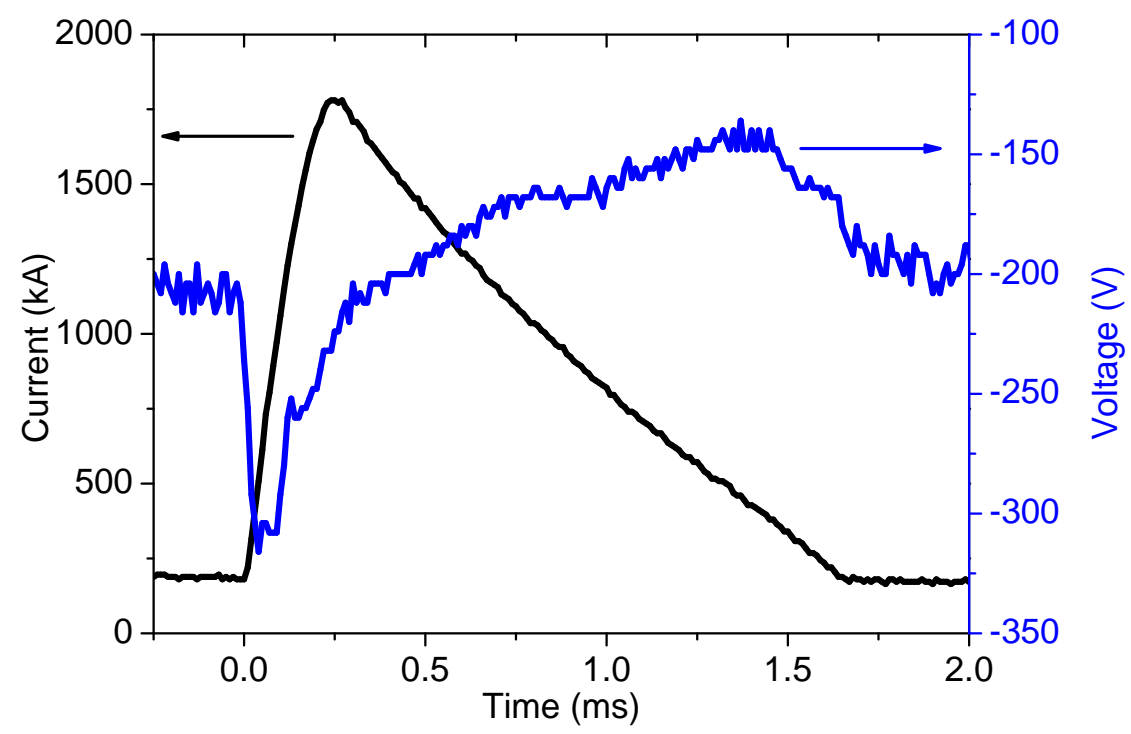

Figure 3: Typical current (dashed) and voltage (solid) trace with a capacitor stored energy of $320 \mathrm{~J}$. The potential was measured between the cathode and the grounded anode of the plasma source, while the current was measured from the capacitor bank with the DC current (190 A) incorporated.

\section{Performance of the pulsed system}

The current and voltage characteristics of the pulses were monitored using a Rogowski coil (Hioki 9667) and voltage probe (LeCroy PPE) respectively. A typical discharge is shown in figure 3. In this case the current rise time for the pulse is $\sim 220 \mu$ s while the e-folding time for the current decay is $\sim 600 \mu \mathrm{s}$. From these IV traces the dissipated power in the source can be established, as shown in figure 4. This demonstrates two trends- firstly that the peak power increases non-linearly with stored energy, while the characteristic decay time $\left(\tau_{\text {decay }}=L_{\text {total }} / R_{\text {total }}\right)$ increases with stored energy following a similar trend. In all cases the rise time is approximately constant at around $210 \pm 10$ $\mu \mathrm{s}$. This is summarised in figure 5 .

By integrating the power evolution over the time of the discharge the energy dissipated in the plasma can be determined and the efficiency of the pulsed source calculated as $\eta=\frac{E_{\text {Dissipated }}}{E_{\text {Stored }}}=92.1 \pm 0.8 \%$ (figure 6) for a single section. This demonstrates that efficiency is constant for all stored energies so that the increasing decay time balances the non-linear increase in peak power. This increase of decay time can be explained through the decreasing resistance of the plasma as a function of input energy. As most of the power is dissipated in the source through Ohmic heating the plasma resistance decreases with increasing electron temperature as [18]

$$
\eta_{e}=2 \times 10^{4}\left(k T_{e}\right)^{-3 / 2} \ln \Lambda
$$




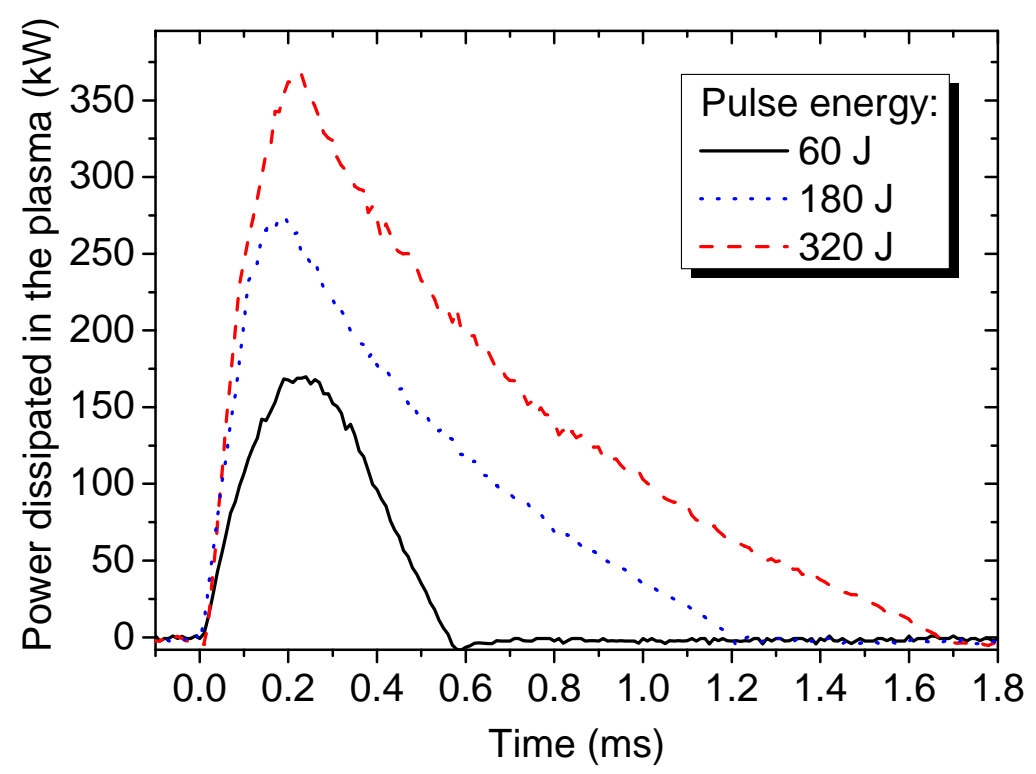

Figure 4: Power evolution dissipated in the plasma as determined from I and V measurements for different stored energies.

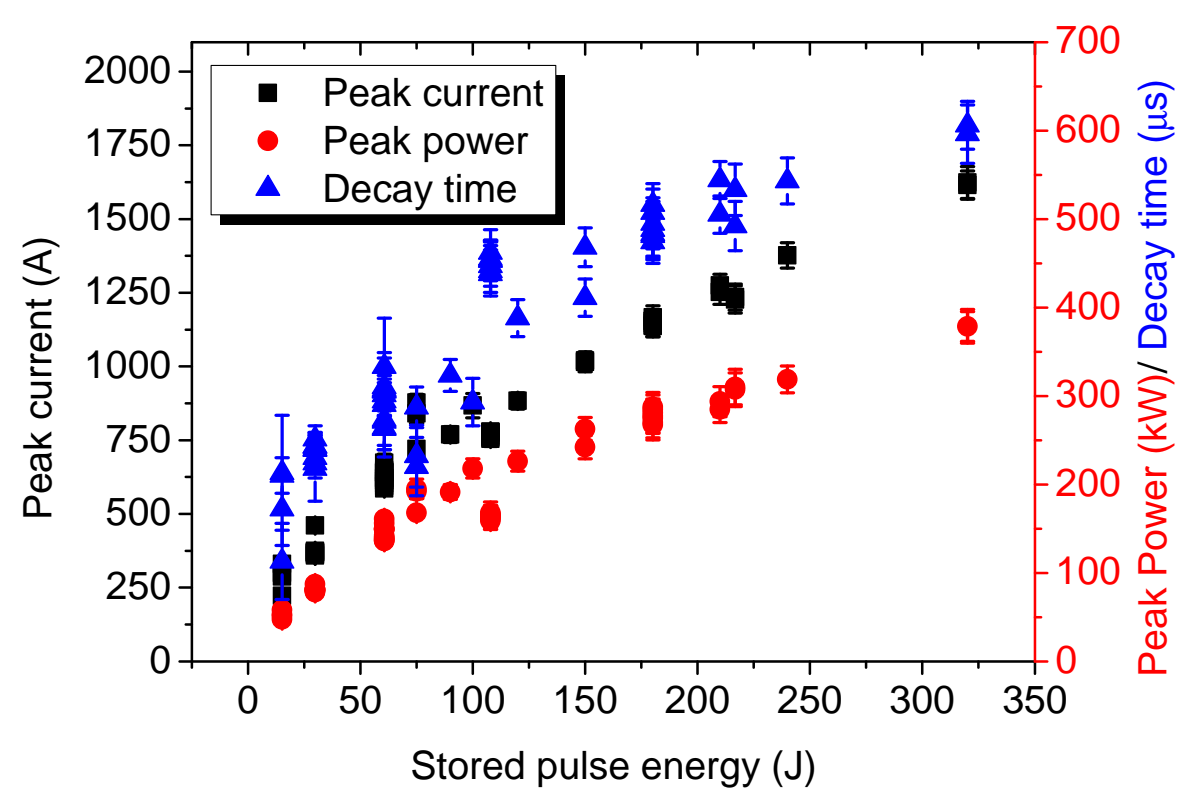

Figure 5: Peak current, peak power and decay time as a function of the stored energy of the pulse, showing all three evolve in a similar non-linear way. 


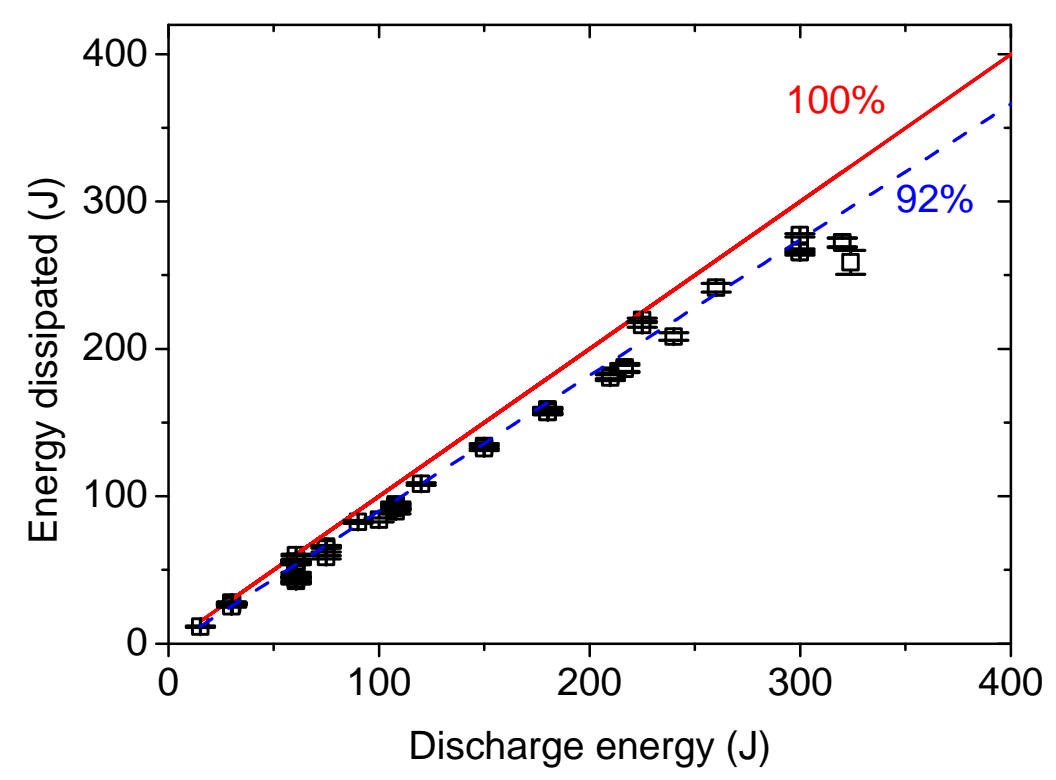

Figure 6: Dissipated energy in the source as a function of capacitor stored energy showing fitted efficiency of $92.1 \%$ and the theoretical maximum of $100 \%$ efficiency.

for a highly ionised hydrogen plasma. The increasing decay time can be fitted as $\tau_{\text {decay }} \propto R_{\text {min }}^{-1.02 \pm 0.02}$ demonstrating that this increase is entirely due to a changing plasma resistivity.

The decay time is defined as by

$$
\tau_{\text {decay }}=\frac{L_{\text {section }} / N+L_{\text {plasma }}}{R_{\text {plasma }}+R_{\text {circuit }}} .
$$

so by adding further capacitor sections the pulse can be shaped. Setting $L_{\text {plasma }}$ and $R_{\text {circuit }}$ to zero gives reasonable agreement, demonstrating that the capacitor inductance is the dominant factor (figure 8). Including them gives values for the plasma inductance of $43.6 \pm 1.6 \mu \mathrm{H}$ and circuit resistance of $0.063 \pm 0.022 \Omega$. While these values are significantly lower and thus less significant than the values of the section inductors and the plasma resistance respectively they still play a small role in determining the circuit response to the discharge.

Examination of the peak power deposited as a function of section number however shows that while initially this leads to a higher peak power, this value rapidly decreases as section number is increased. This is due to the non-perfect efficiency of each section, which leads to a reduced efficiency of the total system as $\eta_{\text {system }}=\eta_{\text {section }}^{N}$ (figure 8). Fitting this to the observed data gives $\eta_{\text {section }}=92 \pm 14 \%$ which is close to the previously measured values. Therefore it is necessary to increase the stored energy in each capacitor to compensate for this effect. 


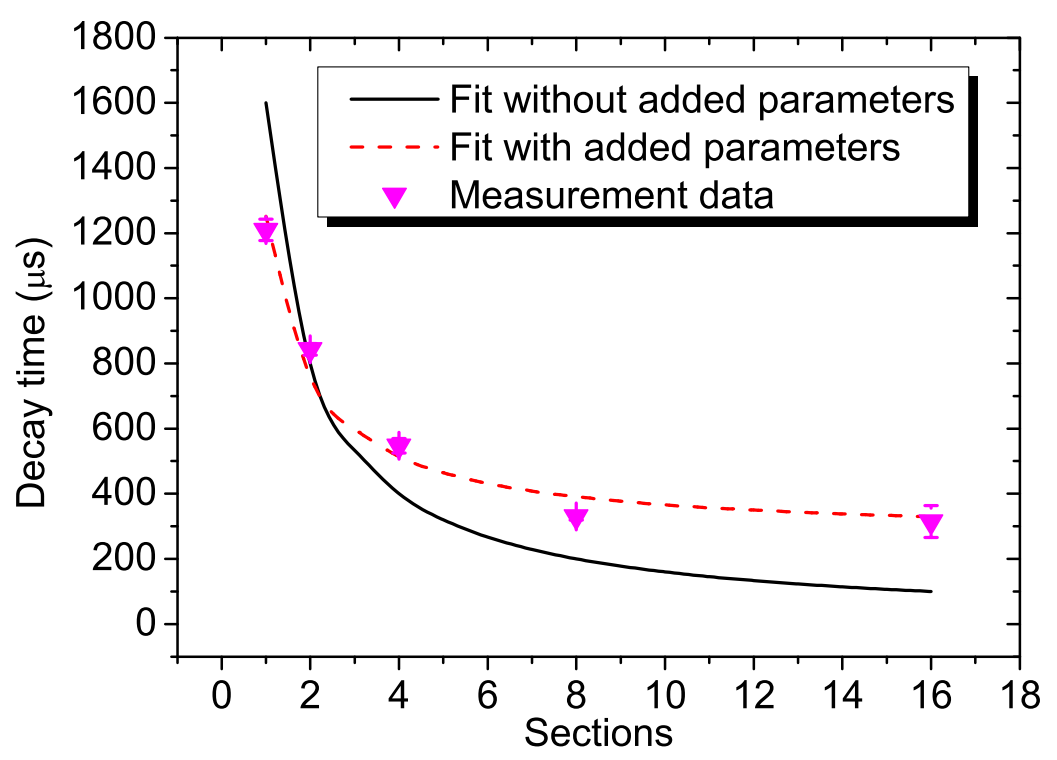

Figure 7: Decay time as a function of capacitor bank section compared against calculated values using the measured minimum resistance for a constant stored energy of $480 \mathrm{~J}$. Also shown are fits to the decay time using either $\tau_{\text {decay }}=L_{\text {section }} / N R_{\text {total }}$ (solid) or equation 4 (dashed). Data was recorded during operation at Pilot-PSI (see section 6)

\section{Evolution of the plasma parameters during a pulse}

Electron density $\left(n_{e}\right)$ and temperature $\left(T_{e}\right)$ of the plasma as a function of beam radius can be determined using a Thomson scattering (TS) system in Magnum-PSI located at a distance $\geq 15 \mathrm{~mm}$ from the target position [19]. The integration time of the TS detection system is much shorter $(\sim 25 \mathrm{~ns})$ than the pulse duration, so that $n_{e}$ and $T_{e}$ can be determined during a particular moment of a pulse within a single integration time. The high electron density during a pulse gives a large signal and thus enables this. By simultaneous triggering of both systems and by utilising a variable delay it is possible to measure a different time interval in the pulse relative to its start. Due to the high replicability of each pulse this can be used to build up a temporal and spatial picture of the pulse through the shot by systematically increasing the delay each time. Typical density and temperature evolutions are shown in figure 9. All measurements were made with an $\mathrm{H}_{2}$ input gas flow rate of $17 \mathrm{~Pa} \mathrm{~m}^{3} \mathrm{~s}^{-1}$, a DC current of $200 \mathrm{~A}$ and a magnetic field in the coil centre of $1.3 \mathrm{~T}$.

During the increased power input to the plasma during the pulse two processes are important. Firstly the temperature of the plasma increases due to Ohmic heating of the electrons, such that the temperature increase closely follows the power trace. Due to this temperature increase the ionization fraction increases dramatically and the electron density quickly reaches a saturated value, consistent with previous observations 


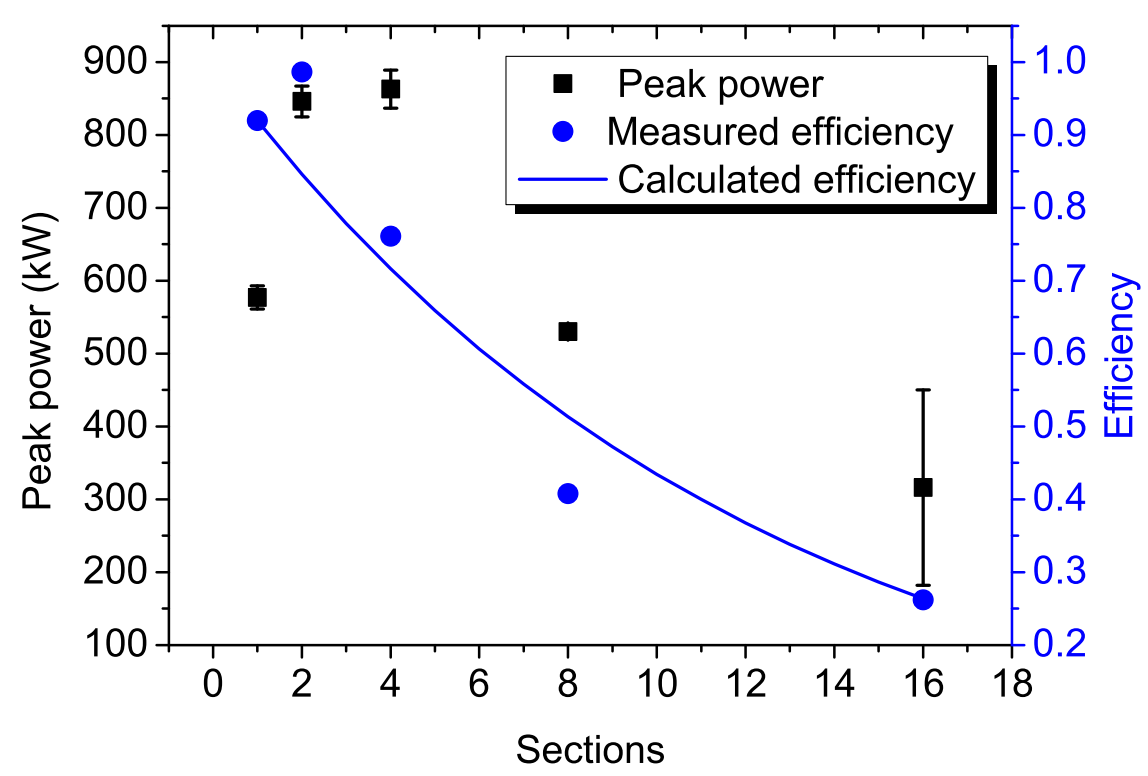

Figure 8: Peak power as a function of number of capacitor sections used for a fixed stored capacitor energy of $480 \mathrm{~J}$. Initially increasing capacitor number leads to an reduced decay time and higher peak power, but as the number of capacitors is increased the efficiency of energy transfer to the plasma is reduced leading to a lower overall peak power. Comparison of the calculated efficiency with the measured values shows reasonable agreement. Data was recorded during operation at Pilot-PSI (see section 6).

of increased electron density with gas flow in Pilot-PSI [20]. An estimation based on the neutral gas flow rate $\left(17 \mathrm{~Pa} \mathrm{~m}^{3} \mathrm{~s}^{-1}\right)$ and the Saha equation for hydrogen gives an ionization fraction $>99 \%$ for $T_{e}>\sim 1.8 \mathrm{eV}$. This agrees well with the high and saturated values for $n_{e}$ observed at temperatures above this. Similarly a precipitous decline in electron density is observed as the temperature falls to low values at the end of the pulse.

In figure $10(\mathrm{a})$ the evolution of the electron temperature at the beam centre $(r=0)$ as a function of time is shown for different stored energies (all pulses use a single section). The pulse length and peak temperature increases in the same manner as the power trace. The temperature at the time of maximum current is plotted in figure 10 (b). For low pulse currents $(<500 \mathrm{~A})$ the temperature increases strongly compared to the DC temperature, but for higher currents this increase becomes incrementally smaller. This is due to the decreasing effect of the Ohmic heating as the resistance decreases, while the electron heat conduction losses increase strongly as a function of the peak electron temperature $\left(Q_{e}^{\text {cond }} \propto T_{e}^{7 / 2}\right)$.

By equating the Ohmic heating $\left(\propto j^{2} / T_{e}^{3 / 2}\right)$ to electron heat conduction losses as in [12] the electron temperature is shown to scale with $T_{e} \propto j^{2 / 5}$. Allometrically fitting this value to the data shows excellent agreement indicating these processes are the dominant 


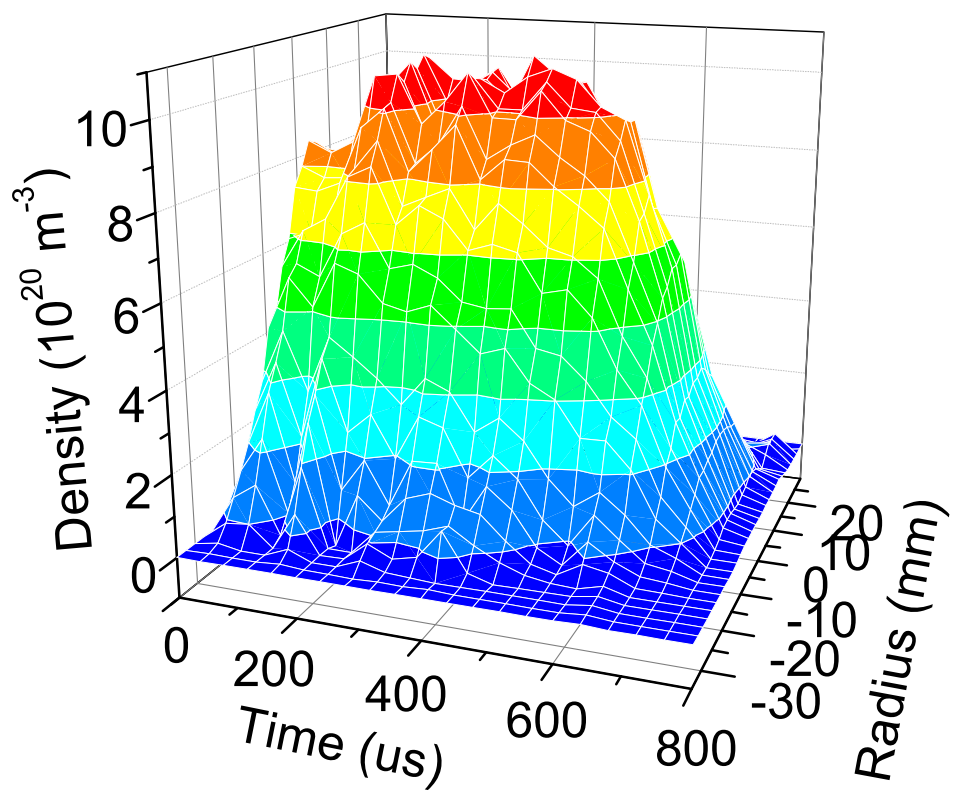

(a)

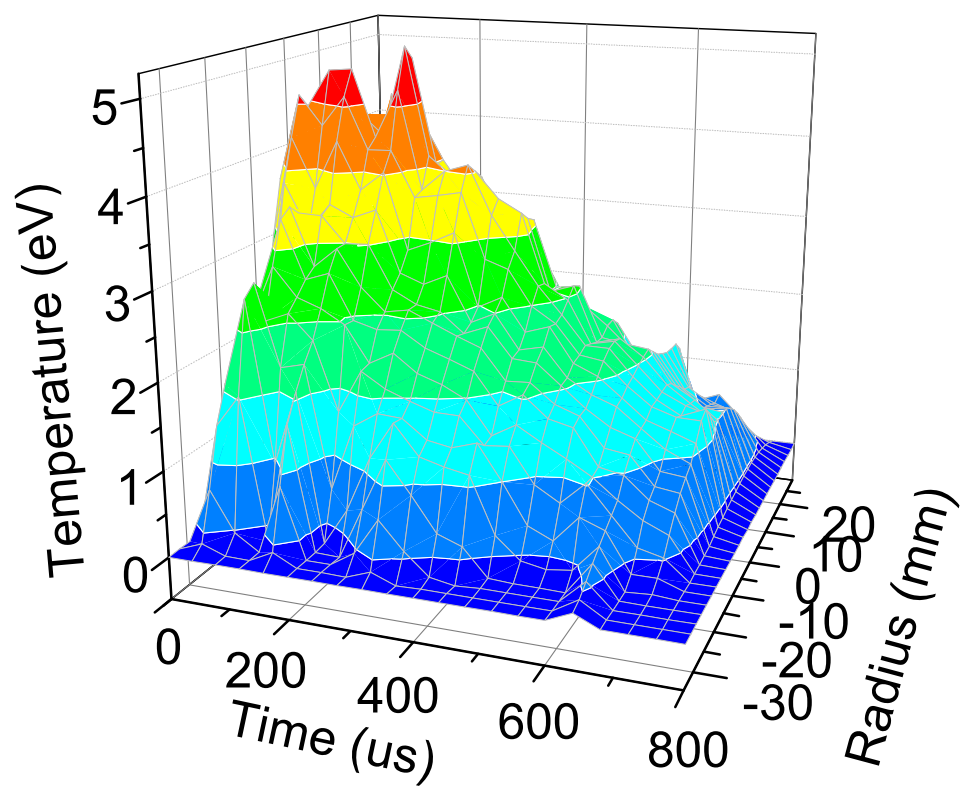

(b)

Figure 9: 3D plots of the temporal and spatial evolution of (a) electron density and (b) electron temperature during a typical $60 \mathrm{~J}$ stored energy plasma pulse, as measured by Thomson scattering $18 \mathrm{~mm}$ in front of the target. These were created using 32 plasma pulses discharged during a single shot with the TS performed at a time interval $25 \mu \mathrm{s}$ earlier each time relative to each pulse. 


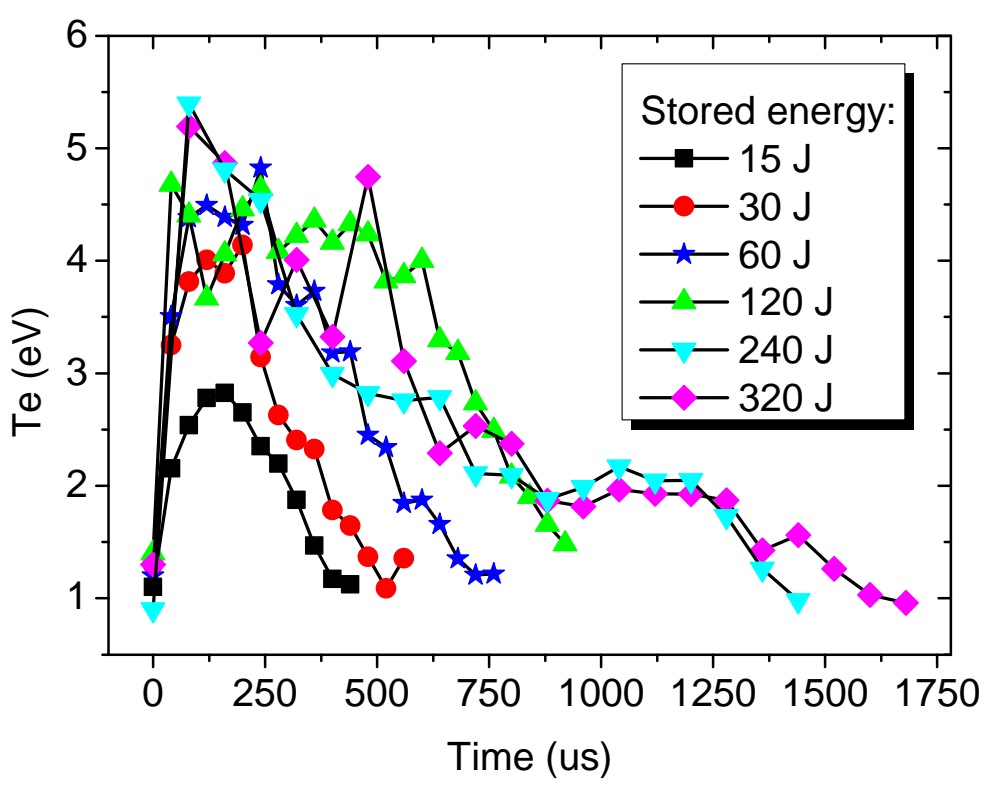

(a)

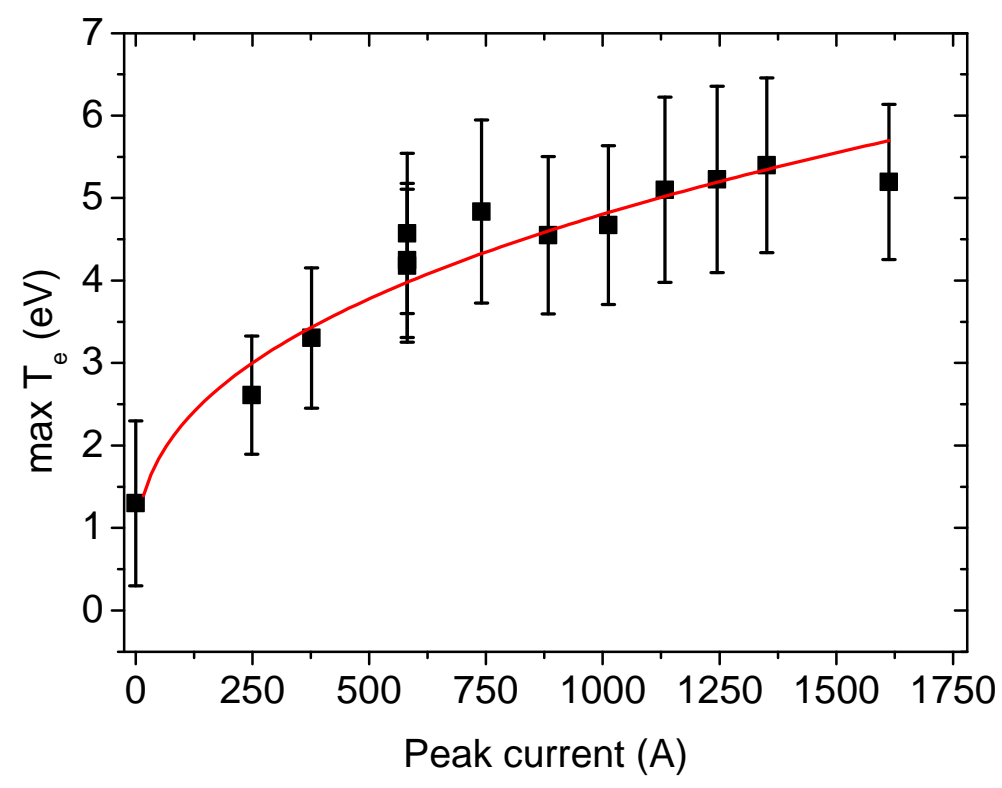

(b)

Figure 10: (a) Evolution of electron temperature at $r=0$ during the pulse for different stored energies utilising a single capacitor bank section. (b) Value of the electron temperature at the time of maximum current (typically $210 \mu$ s after the start of the pulse) as a function of maximum current. The fitted line is of the form $T_{e}=A j^{2 / 5}$ where $A$ is a free fitting parameter. 


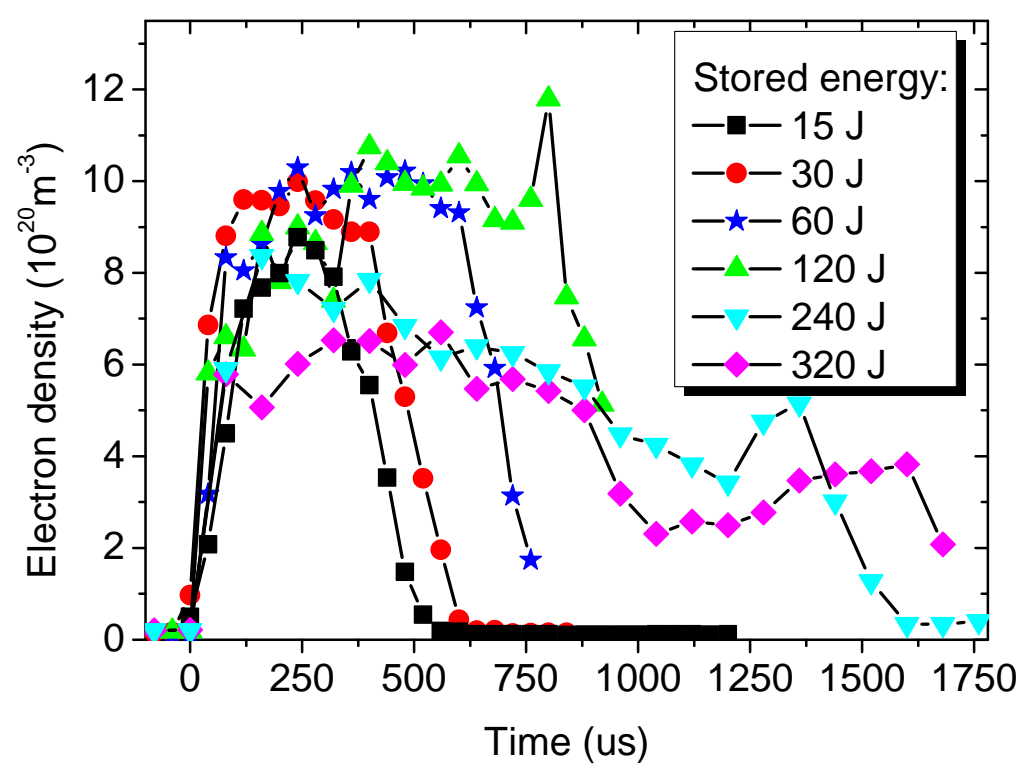

(a)

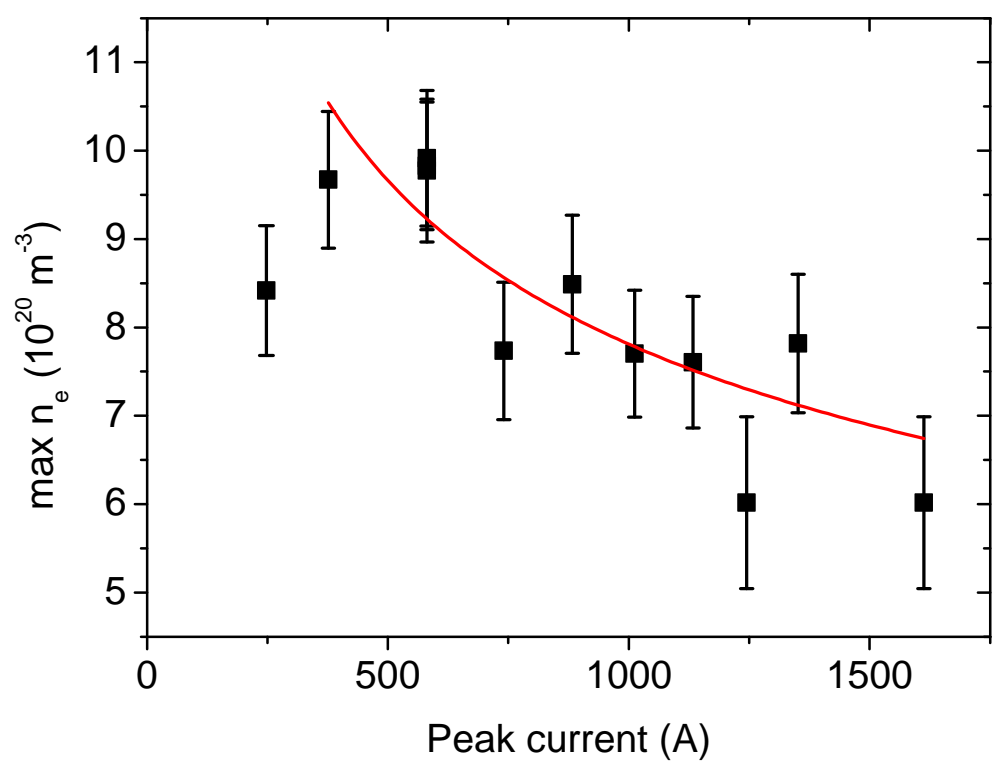

(b)

Figure 11: (a) Evolution of electron density at $r=0$ during the pulse for different stored energies utilising a single capacitor bank section. (b) Value of the electron density at the time of maximum current (typically $210 \mu$ s after the start of the pulse) as a function of maximum current.

ones during the pulse.

Similarly to figure 10 the evolution of the electron density at the beam centre 


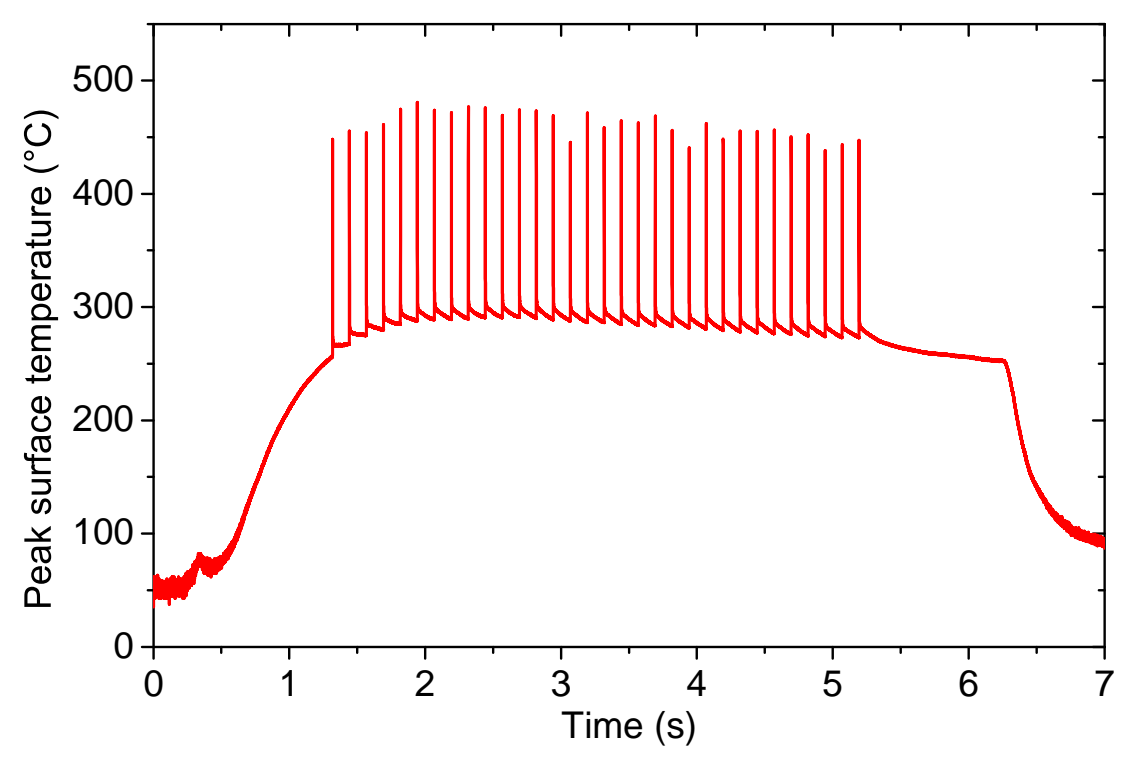

Figure 12: Temperature evolution of a tungsten target at the position $r=0$ subjected to a $200 \mathrm{~A}, 17 \mathrm{~Pa} \mathrm{~m}^{3} \mathrm{~s}^{-1}$ plasma with a $0.9 \mathrm{~T}$ field in the bore of the magnet. $8 \mathrm{~Hz}$ pulses with $55 \mathrm{~J}$ deposited energy were superimposed by capacitor bank discharge.

as a function of time is shown for the same set of pulses in figure 11(a). This demonstrates that the the density is saturated at approximately $1 \times 10^{21} \mathrm{~m}^{-3}$ and that the duration of the saturated density matches well with the time duration of the pulse and with the temperature evolution. As the deposited energy increases, the peak density reaches a lower saturation value. As the total flux is limited by the gas flow input into the source the particle flux $\Gamma=n_{e} v_{t h}$ can be assumed a conserved quantity in the case of full ionization. As the temperature increases the electrons and ions pass the observation position at an increasing average speed and with a larger range of Maxwellian energies, producing a relative rarefaction of the plasma pulse. The maximum probability distribution for the speed decreases as a function of temperature as $T_{e}^{-1 / 2}$. Therefore the maximum electron density should decrease with current as $j^{-1 / 5}$. Figure 11 shows the peak value of $n_{e}$ at the time of maximum current. The data where density saturation has been reached $\left(E_{\text {stored }}>15 \mathrm{~J}\right)$ is allometrically fitted and shows a power dependence of $-0.307 \pm 0.062$ which therefore shows reasonable agreement within the fitting error.

\section{Evolution of the target parameters during a pulse}

The increased plasma density and temperature in turn increase the surface temperature of the target transiently each time. A temperature trace as recorded using the FLIR infra-red camera is shown in figure 12 (a) for a typical discharge. Here the magnetic field is turned on at $t=0 \mathrm{~s}$ leading to a surface temperature increase up to $\sim 480$ 
${ }^{\circ} \mathrm{C}$. The slight decline in baseline temperature through the discharge after the initial heating phase originates from a slow decrease of the magnetic field strength of the coils due to their heating (and also limits the duration of the discharge). Each firing of the capacitor leads to an average temperature increase of $173 \pm 8^{\circ} \mathrm{C}$ each time. Heat fluxes can be determined from the temperature evolution using the numerical heat flux solver THEODOR [21]. This solves the heat flux equation in two-dimensions for a flat surface

$$
\rho C \frac{\partial T(x, t)}{\partial t}=\nabla(k \nabla T(x, t))
$$

from the temperature distribution $T(x, t)$ across the surface $x$, with $\rho, C$ and $k$ the density, heat capacity and thermal conductivity of the bulk material respectively. A more thorough description of the code is given in [22]. Heat transfer coefficients on the top $\left(\alpha_{t o p}\right)$ and bottom $\left(\alpha_{\text {bottom }}\right)$ of the sample act as boundary conditions to be optimized for and are given by

$$
q_{\text {plasma }}=\alpha_{\text {top }}\left(T_{I R}-T_{\text {bulk }}\right)
$$

and

$$
q_{\text {cooling }}=\alpha_{\text {bottom }}\left(T_{\text {bulk }}-T_{\text {cooling }}\right) .
$$

$\alpha_{\text {bottom }}$ was determined by iteratively minimising the heat flux after the discharge to zero (and has little effect upon the transient heat flux determination), while $\alpha_{\text {top }}$ was found using the methodology described in [23], leading to $\alpha_{\text {bottom }}=80 \mathrm{~kW} \mathrm{~m}^{-2} \mathrm{~K}^{-1}$ and $\alpha_{\text {top }}=0.8 \mathrm{MW} \mathrm{m}^{-2} \mathrm{~K}^{-1}$. The value for $\alpha_{\text {top }}$ represents the effects of surface roughness producing a thin layer of reduced conductivity at the surface and is required to avoid negative heat fluxes after the transients [24 26. Analysis of the same discharges with the ANSYS package using finite element analysis of the full 3D geometry of the target [27] demonstrates that indeed a thin barrier layer is required to avoid this effect.

The temperature increase of a tungsten sample and the plasma heat flux at $r=0$ as a function of peak current is shown in figure 13 . As the ramp-time of the pulse is approximately constant in all cases the relationship between temperature and heat flux is a linear one and therefore both follow the same form as the source current increases, as $P \sqrt{t_{\text {rise }}} \propto \Delta T[28]$. The heat flux to the surface can also be defined as

$$
q=\gamma k_{B} T_{e} \Gamma_{s e} \approx\left(\frac{2}{3 m_{i}}\right)^{1 / 2} \gamma n_{e}\left(k_{B} T_{e}\right)^{3 / 2}
$$

where $\gamma$ is the sheath heat transmission coefficient (incorporating contributions from ions), $\Gamma_{s e}$ is the ion flux to the surface and $m_{i}$ the ion mass, and the specific heat ratio is assumed to be $5 / 3$ [29]. From this figure 13 can be understood from the evolution of electron density and temperature as a function of current. The initial strong increase in heat flux for currents below $600 \mathrm{~A}$ results from the combined increase in both $n_{e}$ and $T_{e}$. Between 600 and $1250 \mathrm{~A}$ the heat flux values fall as the values of $n_{e}$ fall sharply in this range due to the saturation in ionization and pulse rarefaction. For values $>1250$ A the temperature increase (scaling in equation 8 as $\propto \sim I^{3 / 5}$ ) overcomes the decrease 


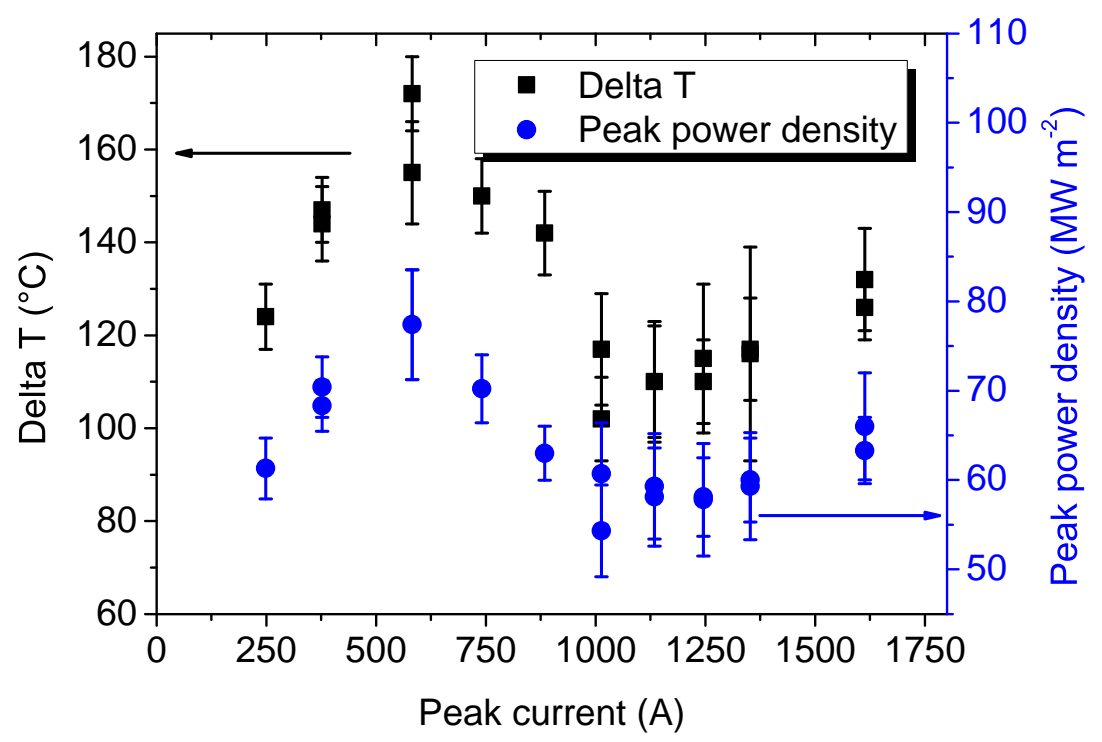

Figure 13: Peak temperature increase (squares) and maximum heat flux to the surface as determined from the THEODOR code (circles) due to a transient pulse as a function of peak input current to the source. Pulses range from $15 \mathrm{~J}$ to $320 \mathrm{~J}$ stored energy.

in $n_{e}$ (scaling as $\propto \sim I^{-1 / 5}$ ). Therefore the heat flux should scale with $\sim I^{2 / 5}$ for large currents. Allometric scaling of the form $q=A I^{b}$ gives the power dependence with respect to current as $b=0.42 \pm 0.04$ in the range 1250 to $1600 \mathrm{kA}$ which is in good agreement with this.

As time resolved Thomson scattering measurements are available for these measurements these provide a way to determine the value of the sheath heat transmission factor $\gamma$. In figure 14 measured heat flux is compared with the expected values of heat flux to the surface $(q)$ as calculated from equation 8 for two example pulse conditions. The values of $n_{e}$ and $T_{e}$ as determined in section 4 were used while keeping $\gamma$ as a free parameter. This calculation shows good agreement in the shape of the pulse in comparison with the THEODOR calculation and gives a value $\gamma \approx 7.7$ for all compared pulses. From sheath theory $\gamma$ can be calculated as in [30] (where we assume $T_{i} \approx T_{e}$ based on Doppler broadening of Balmer- $\beta$ (486.1 nm) emission [12, 31]). For the electron temperature range of 2.8-5.9 eV (the range of measured values), the values of $\gamma$ decreases from 9.3 to 7.3 indicating these results are consistent with expectations.

\section{Discussion}

The measured heat fluxes using MERS are significantly lower than those measured in Pilot-PSI using the original capacitor bank system where heat fluxes up to $1.2 \mathrm{GW}$ $\mathrm{m}^{-2}$ were achieved [11]. These values were achieved with a source current of $\sim 12 \mathrm{kA}$ however, while the maximum investigated here was $1.7 \mathrm{kA}$ to ensure the good lifetime of 


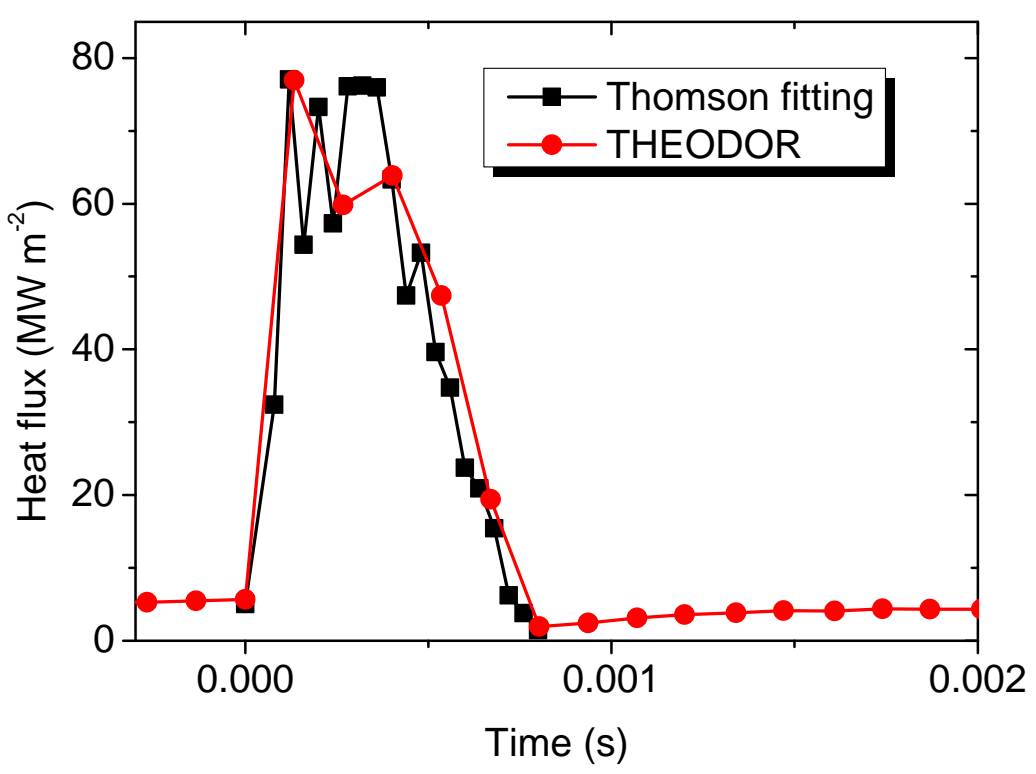

(a)

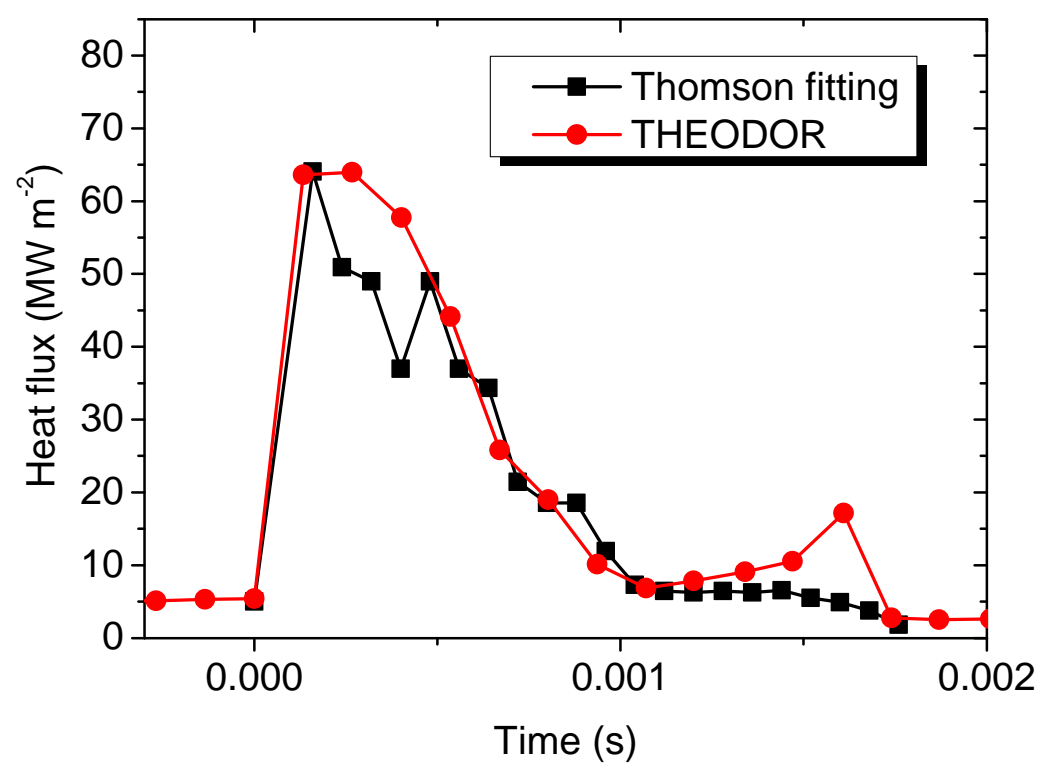

(b)

Figure 14: Evolution of the heat flux at $r=0$ calculated either from equation 8 with $\gamma=7.7$ (squares) or from the THEODOR code (circles) for pulses with either (a) $60 \mathrm{~J}$ stored energy or (b) $320 \mathrm{~J}$ stored energy. 


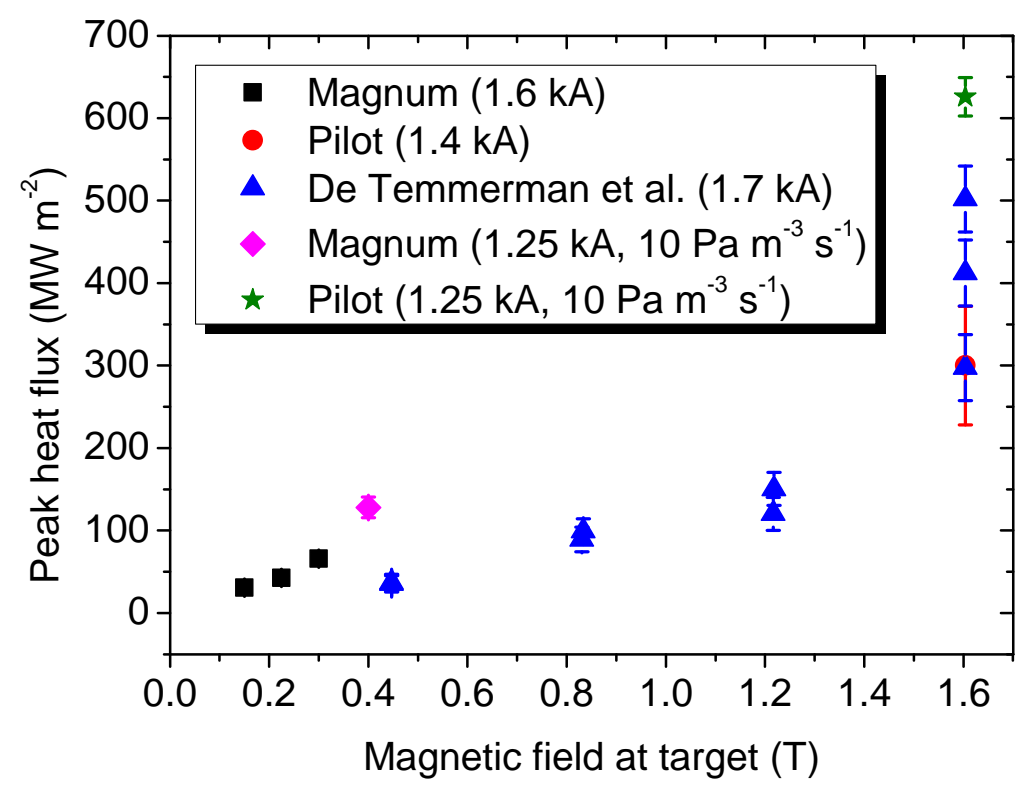

Figure 15: Peak heat flux measured at $r=0$ as a function of magnetic field at the target position in either Magnum-PSI using MERS by changing the overall magnetic field strength (squares), or by moving the target towards the source (diamonds) or in Pilot-PSI using both the single-pulse system (triangles) or MERS (circles and stars). The single-pulse results are from De Temmerman et. al. (2010) 32

the plasma source (as the MERS system aims at investigating the effect of large numbers of ELM-like pulses on material surfaces). A second reason for the lower heat flux is that the magnetic field of Magnum-PSI diverges beyond the position of the solenoids, while the target is located $0.6 \mathrm{~m}$ from the exit of the coils. Therefore at the target position the maximum value of the magnetic field $\left(B_{t}\right)$ is $0.3 \mathrm{~T}$ rather than $1.3 \mathrm{~T}$ within the coil region $\left(B_{c}\right)$. Due to this expansion, from the frozen-in condition and Gauss' law, the beam size is likewise expanded, i.e. $B_{c} A_{c}=B_{t} A_{t}$. From particle conservation the particle flux density is thus reduced, leading to a reduction in heat flux to the target (equation 8). We can describe this as

$$
\frac{q_{t}}{B_{t}}=\frac{q_{c}}{B_{c}} .
$$

To investigate this effect further the Magnum-PSI magnetic field was varied from 0.15 to $0.3 \mathrm{~T}$ at the target position (by varying the coil current) utilising a capacitor stored energy of $320 \mathrm{~J}$, leading to a peak source current of $1.6 \mathrm{kA}$. This lead to a doubling of the heat flux to the surface as expected from equation 9 (figure 15). These values can be compared with those measured in Pilot-PSI with the single-pulse system [32] as the source current used was similar $(1.7 \mathrm{kA})$ and plasma conditions were identical in gas flow and DC current supply. This shows that the heat flux values are comparable, 
while being approximately two times higher than those that can be extrapolated from the Pilot data for a similar magnetic field strength. However, in [32] the source channel diameter was smaller which may account for the difference.

To determine any differences in the performance of MERS and the single-pulse capacitor bank system MERS was coupled to Pilot-PSI using an identical source. The pulse currents and voltages were compared to those measured in Magnum-PSI and found to be extremely similar, indicating that within the source the performance of the system is identical in either machine. As a more complete data-set was available from Pilot experiments the I-V characteristics recorded were used in section 3 where indicated. Heat flux measurements in Pilot-PSI using MERS with a source current of $1.4 \mathrm{kA}$ and a magnetic field of $1.6 \mathrm{~T}$ are also shown in figure 15 and show good agreement with the earlier Pilot-PSI data.

Reducing the gas flow in the source has the effect of reducing the electron density and increasing the electron temperature of the plasma exiting the source 33 due to the lower number of hydrogen atoms to be ionized and thus higher energy per atom available to heat it. As the heat flux density is a stronger function of temperature than density an inversely proportional change can elicit a stronger heating effect. Reducing the gas flow rate also reduces the background pressure in the vessel which in turn reduces molecular assisted recombination (MAR) which is strongly pressure dependent [34]. As MAR is a most effective recombination mechanism for electron temperatures between 0.5 and $2 \mathrm{eV}$ [35] for the pulse MAR plays a role only in the edges of the beam, but some reduction in recombination is expected. To attempt to utilise these two effects the input gas flow in Pilot was reduced from $17 \mathrm{~Pa} \mathrm{~m}^{3} \mathrm{~s}^{-1}$ to $10 \mathrm{~Pa} \mathrm{~m}^{3} \mathrm{~s}^{-1}$ and thus the background pressure from 10 to $6 \mathrm{~Pa} \mathrm{~m}^{3} \mathrm{~s}^{-1}$. In this case the heat flux deposited was increased to $626 \pm 23 \mathrm{MW} \mathrm{m}^{-2}$, even in the non-optimised case of $1.25 \mathrm{kA}$ peak current. As time-resolved Thomson scattering, as described in section 4, was not available it was not possible to distinguish the contributions from changing temperatures and electron densities compared with reduced recombination however.

To combine these effects with increasing the magnetic field at the target in MagnumPSI the position of the target was moved towards the source (beyond the Thomson scattering position) so that the magnetic field at the target position increased to $0.4 \mathrm{~T}$ from $0.3 \mathrm{~T}$, while the gas flow was decreased from $17 \mathrm{~Pa} \mathrm{~m}^{3} \mathrm{~s}^{-1}$ to $10 \mathrm{~Pa} \mathrm{~m}^{3} \mathrm{~s}^{-1}$ (a reduction in background pressure from $0.5 \mathrm{~Pa}$ to $0.35 \mathrm{~Pa}$ ). In this case the heat flux increased to $128 \pm 12 \mathrm{MW} \mathrm{m}^{-2}$ at a slightly lower input current of $1.25 \mathrm{kA}$, consistent with the expected scaling. As Magnum-PSI is due to be upgraded to a super-conducting magnet which will have a field at the target position of up to $2.5 \mathrm{~T}$ [36] this would translate to a heat flux in Magnum-PSI of $\sim 550 \mathrm{MW} \mathrm{m}^{-2}$ for a $1.6 \mathrm{kA}$ pulse at a gas flow of $17 \mathrm{~Pa} \mathrm{~m}^{3} \mathrm{~s}^{-1}$ or approximately $950 \mathrm{MW} \mathrm{m}^{-2}$ at $10 \mathrm{~Pa} \mathrm{~m}^{3} \mathrm{~s}^{-1}$. A heat flux of several $\mathrm{GW} \mathrm{m}^{-2}$ would be possible if the power dependence observed in section 5 for high input currents continues at higher values, but this is contingent upon the performance and survival of the source under these conditions. So far the upper current limit for good coating lifetime is yet to be reached which suggests higher heat fluxes 
should be achievable.

Upgrading to this higher and straight magnetic field will produce a more realistic replication of ITER-like ELMs in terms of heat and particle fluxes than has previously been achieved in Pilot-PSI or Magnum-PSI. Differences which will be expected to remain are that the transient heating profile could be narrower, which would lead to higher thermal shock stresses, and that the ion energies will be lower in Magnum-PSI than at the ITER divertor strikepoints.

Predictions from RACLETTE show that at the inboard side of the divertor outer strikepoint the HWHM of the temperature profile will be around $20 \mathrm{~mm}$ in steady state [37, while any footprint broadening expected for ELMs is not certain [8]. However, the $\lambda_{q}$ used in [37] was $5 \mathrm{~mm}$, while recent scaling law calculations predict $\lambda_{q}$ could be as low as $1 \mathrm{~mm}$ [38] which would likely reduce this HWHM value. In Magnum-PSI the HWHM will be around $5 \mathrm{~mm}$, so that the thermal gradients may be somewhat higher for large spreading, but nonetheless of similar magnitude. By tilting the target the power can be spread to match the ITER divertor thermal gradients if this effect is crucial, although at the cost of reduced heat flux.

The ion energies expected in ITER are $\sim 100 \mathrm{eV}$ during type-I ELMs, while those produced using the MERS system in this case are expected to be around $10-15 \mathrm{eV}$. To improve on this situation a pulsed bias system will be installed which will permit transient biasing up to $-1000 \mathrm{~V}$ in combination with the pulsed source and thus reach much higher ion energies. This should also lead to an increase in heat flux which will contribute towards reaching the ITER-relevant ELM regime.

\section{Conclusion}

A Multiple-pulse ELM Replication System has been successfully constructed for the Magnum-PSI and Pilot-PSI linear plasma devices. This transiently delivers a highly increased current to the source by discharging a set of coupled capacitor banks which lead to Ohmic heating and increased ionization of the plasma. A single pulse Thomson scattering measurement mode was implemented in Magnum-PSI to determine the transient increase in electron density and temperature at the target position of up to $1 \times 10^{21} \mathrm{~m}^{-3}$ and $5 \mathrm{eV}$ respectively for a time period $\sim 0.5-1.5 \mathrm{~ms}$. This system therefore combines both a greatly increased particle and heat flux to the target which well replicates the conditions occurring during an ELM-pulse in the divertor region.

Heat fluxes to a tungsten surface were determined using the THEODOR code to be up to $\sim 130 \mathrm{MW} \mathrm{m}^{-2}$ for a peak pulse current of $1250 \mathrm{~A}$ in the source in MagnumPSI. The time evolution of the heat flux agreed well with that determined using the Thomson scattering measurements. From these results a value for the sheath heat transmission factor was determined, and found to be $\gamma \approx 7.7$, which agrees well with values calculated from sheath theory. The surface heat flux was found to be strongly dependent on magnetic field strength at the target position and on the source gas flow rate, and heat fluxes of more than $600 \mathrm{MW} \mathrm{m}^{-2}$ were found when the MERS system was 
used in a 1.6 T field in Pilot-PSI under similar experimental conditions to Magnum-PSI. Extrapolation to the fields expected after installation of a high-field superconducting magnet in Magnum-PSI indicates that heat fluxes in the $\mathrm{GW} \mathrm{m}^{-2}$ region should be reached.

\section{Acknowledgments}

This work is part of the research program of the Stichting voor Fundamenteel Onderzoek der Materie (FOM), which is financially supported by the Nederlandse Organisatie voor Wetenschappelijk Onderzoek (NWO). It is supported by the European Communities under the contract of Association between EURATOM and FOM and carried out within the framework of the European Fusion Program, and of the European Taskforce on PlasmaWall Interactions.

\section{References}

[1] Janeschitz G, Borrass K, Federici G, Igitkhanov Y, Kukushkin A, Pacher H D, Pacher G and Sugihara M 1995 J. Nucl. Mater. 220-222 73-88

[2] ITER Physics Expert Group on Divertor et al. 1999 Nucl. Fusion 392391

[3] Loarte A et al. 2007 Nucl. Fusion 47 S203-S263

[4] Baldwin M J and Doerner R P 2010 J. Nucl. Mater. 404(3) 165-173

[5] Kajita S, Yoshida N, Yoshihara R, Ohno N and Yamagiwa M 2011 J. Nucl. Mater. 418(1-3) 152-158

[6] Miyamoto M, Nishijima D, Ueda Y, Doerner R, Kurishita H, Baldwin M J, Morito S, Ono K and Hanna J 2009 Nucl. Fusion 49(6) 065035

[7] Alimov V K, Shu W M, Roth J, Sugiyama K, Lindig S, Balden M, Isobe K and Yamanishi T 2009 Phys. Scr. T138 014048

[8] Loarte A et al. 2007 Phys. Scr. T128 222-228

[9] Kajita S, Ohno N, Takemure S, Sakaguchi W and Nishishima D 2007 Appl. Phys. Lett. 91261501

[10] Umstadter K R, Doerner R and Tynan G 2009 J. Nucl. Mater. 386-388 751-755

[11] De Temmerman G, Zielinski J J, van Diepen S, Marot L and Price M 2011 Nucl. Fusion 51073008

[12] Zielinski J J, van der Meiden H J, Morgan T W, Schram D C and De Temmerman G 2012 Plasma Sources Sci. Technol. 21065003

[13] Maecker H 1956 Z. Naturforsch. 11(A) 457

[14] van der Sanden M C M, Janssen G M, de Regt J M, Schram D C and van der Mullen J A M 1992 Rev. Sci. Instrum. 633369

[15] van Eck H J N, Kleyn A W, Lof A, van der Meiden H J, van Rooij G J, Scholten J and Zeijlmans van Emmichoven P A 2012 Appl. Phys. Lett. 101224107 
[16] Scholten J et al. 2013 Fusion Eng. Des. 88 1785-1788

[17] De Temmerman G et al. 2013 Fusion Eng. Des. 88 483-387

[18] Spitzer L 1956 Physics of Fully Ionized Gases (New York: Interscience)

[19] van der Meiden H J et al. 2012 Rev. Sci. Instrum. 83(12) 123505

[20] Zielinski J J, Al R, van der Meiden H, Melissen W, Rapp J and De Temmerman G 2011 J. Nucl. Mater. 415 S70-S73

[21] Herrmann A, Junker W, Gunther K, Bosch S, Kaufmann M, Neuhauser J, Pautasso G, Richter T and Schneider R 1995 Plasma Phys. Control. Fusion 37(1) 17

[22] Greuner H, Böswirth B, Eich T, Herrmann A, Maier H and Sieglin B 2014 Phys. Scr. T159 014003

[23] Eich T, Andrew P, Herrmann A, Fundamenski W, Loarte A, Pitts R A and JETEFDA contributors 2007 Plasma Phys. Control. Fusion 49573604

[24] Herrmann A 2001 28th EPS Conference on Controlled Fusion and Plasma Physics (Funchal, Portugal)

[25] Herrmann A, Balden M, Bohmeyer W and Hildebrandt D 2004 Phys. Scr. T111 98-100

[26] Delchambre E, Counsell G and Kirk A 2009 Plasma Phys. Control. Fusion 51 055012

[27] 2013 ANSYS Academic Research, Release 14.5.7

[28] Carslaw H S and Jaeger J C 1959 Conduction of heat in solids, 2nd Ed. (London: Oxford University Press)

[29] Stangeby P C 2000 The plasma boundary of magnetic fusion devices (Bristol: IOP Publishing)

[30] van den Berg M, Bystrov K, Pasquet R, Zielinski J and De Temmerman G 2013 J. Nucl. Mater. 438 S431-S434

[31] Shumack A E, Veremiyenko V P, Schram D C, de Blank H J, Goedheer W J, van der Meiden H J, Vijvers W A J, Westerhout J, Lopes Cardozo N J and van Rooij G J 2008 Phys. Rev. E 78046405

[32] De Temmerman G, Zielinski J J, van der Meiden H, Melissen W and Rapp J Appl. Phys. Lett. 97

[33] Zielinski J J 2013 A high power pulsed plasma system for material testing under simultaneous continuous and transient loads, http://alexandria.tue.nl/extra2/748465.pdf Ph.D. thesis Eindhoven University of Technology, The Netherlands

[34] Ohno N, Ezumi N, Takamura S, Krasheninnikov S I and Pigarov A Y 1998 Phys. Rev. Lett. 81(4) 818-821

[35] Yu Pigarov A 2002 Phys.Scr. T96 16-31

[36] Rapp J et al. 2010 Fusion Eng. Des. 85 1455-1459 
[37] Pitts R et al. 2013 J. Nucl. Mater. 438 S48-S56

[38] Eich T et al. 2013 Nucl. Fusion 53093031 